\title{
H3-3A Gene
}

National Cancer Institute

\section{Source}

National Cancer Institute. H3-3A Gene. NCI Thesaurus. Code C101443.

This gene is involved in chromatin structure. 